\title{
NONBIJECTIVE IDEMPOTENTS PRESERVERS OVER SEMIRINGS
}

\author{
MARKo OREL
}

\begin{abstract}
We classify linear maps which preserve idempotents on $n \times n$ matrices over some classes of semirings. Our results include many known semirings like the semiring of all nonnegative integers, the semiring of all nonnegative reals, any unital commutative ring, which is zero divisor free and of characteristic not two (not necessarily a principal ideal domain), and the ring of integers modulo $m$, where $m$ is a product of distinct odd primes.
\end{abstract}

\section{Introduction}

Linear preserving problems is an active research area in matrix and operator theory. It concerns with classification of linear maps which preserve some functions, subsets, relations, etc. One of these invariants, preservers of which were already studied by many mathematicians, is the set of all idempotents. We refer to $[1,3,5,12]$ for linear maps which preserve idempotents on $n \times n$ matrices over fields and rings. Linear maps which strongly preserve idempotents (i.e., $A$ is idempotent if and only if $\Phi(A)$ is idempotent) on matrices over antinegative semirings, which are zero divisor free, were studied in [2]. In particular, a complete classification was obtained for the semiring of all nonnegative integers, the semiring of all nonnegative reals, chain semiring, and for binary Boolean algebra. The last result was later generalized to arbitrary finite Boolean algebra [11, Theorem 3.2], which is a semiring isomorphic to a direct product of binary Boolean algebras. Very recently, similar problems as in [2] and [11, Theorem 3.2] were studied in [13, 7]. Here, linear maps $\Phi$ were not assumed to preserve idempotents strongly (i.e., $A^{2}=A$ implies $\Phi(A)^{2}=\Phi(A)$ but not vice versa). However, it was assumed that the maps $\Phi$ were bijective. On the contrary, in the case of matrices over fields $[1,5]$, neither bijectivity nor strong preserving of idempotents was assumed (see also [12]). In fact, for fields of characteristic different from 2 , the semigroup of nonzero linear maps

Received October 1, 2008.

2000 Mathematics Subject Classification. 15A04, 15A33, 16Y60.

Key words and phrases. linear preserver, semiring, idempotent matrix.

This work was supported by grants from the Ministry of Higher Education, Science and Technology, Slovenia. 
that preserve idempotents is generated by transposition and similarity. Hence, any such map is automatically bijective and strongly preserves idempotents. Is it possible to obtain a result, similar to $[1,5]$, also for matrices over semirings? Our main results answer to this question positively for two considerably large classes of semirings. The first class consist of commutative multiplicatively cancellative semirings, which are not antinegative, and such that $2 \neq 0$. The second class is formed by additively cancellative antinegative semirings, which are zero divisor free. As a corollary a complete classification of linear idempotents preservers is obtained for commutative semirings, which are additively and multiplicatively cancellative, and such that $2 \neq 0$. For more details see Theorem 2.1, Theorem 2.3, Corollary 2.5, and Corollary 2.6.

The rest is organized as follows. In Section 2 we recall the necessary definitions and state the main results of this paper. In Section 3 the proofs are given while in Section 4 the analogous problem for a direct product of semirings is considered. Some interesting examples of semirings which fit the main theorems are given in Section 5. In this section we also list counterexamples which show that various assumptions in the main theorems cannot be omitted.

\section{Preliminaries and statements of main results}

A semiring $\mathbb{S}$ consists of a set and two binary operations, addition $(+)$ and multiplication $(\cdot)$, such that:

(a) $(\mathbb{S},+)$ is a commutative monoid with identity element 0 ;

(b) $(\mathbb{S}, \cdot)$ is a monoid with identity element $1 \neq 0$;

(c) multiplication is distributive over addition on both sides;

(d) $s 0=0=0 s$ for all $s \in \mathbb{S}$.

The multiplication symbol is usually omitted, i.e., st $:=s \cdot t$. A semiring $\mathbb{S}$ is called:

- commutative $(\mathrm{COM})$ if the monoid $(\mathbb{S}, \cdot)$ is commutative;

- antinegative $(\mathrm{AN})$ if $s+t=0$ implies $s=0=t$ for any $s, t \in \mathbb{S}$;

- additively cancellative (AC) if $s+t=s+u$ implies $t=u$ for any $s, t, u \in \mathbb{S}$;

- multiplicatively cancellative from left (MCL) if $s t=s u, s \neq 0$ imply $t=u$ for any $s, t, u \in \mathbb{S}$;

- multiplicatively cancellative from right (MCR) if $t s=u s, s \neq 0$ imply $t=u$ for any $s, t, u \in \mathbb{S}$;

- multiplicatively cancellative (MC) if it is MCL and MCR;

- zero divisor free (ZDF) if $s t=0$ implies $s=0$ or $t=0$ for any $s, t \in \mathbb{S}$.

It is easy to see that each of the properties MC, MCL, or MCR implies ZDF, but not conversely (see e.g. Counterexample 5.7).

A map $\varphi: \mathbb{S} \rightarrow \mathbb{S}^{\prime}$ between two semirings is called a semiring homomorphism if $\varphi(s+t)=\varphi(s)+\varphi(t)$ and $\varphi(s t)=\varphi(s) \varphi(t)$ holds for all $s, t \in \mathbb{S}$, and if in addition $\varphi(0)=0$ and $\varphi(1)=1$. When it is bijective, it is called a semiring isomorphism. The center of a semiring $\mathbb{S}$, i.e., the set $\{s \in \mathbb{S} \mid s t=t s \forall t \in \mathbb{S}\}$, 
is denoted by $Z(\mathbb{S})$. Let $M_{n}(\mathbb{S})$ denote the set of all $n \times n$ matrices with entries from $\mathbb{S}$. A matrix with 1 at position $(i, j)$ and zeros elsewhere is denoted by $E_{i j}$, while $I$ denotes the identity matrix. A matrix $A \in M_{n}(\mathbb{S})$ is called idempotent if $A^{2}=A$. A map $\Phi: M_{n}(\mathbb{S}) \rightarrow M_{n}(\mathbb{S})$ preserves idempotents if $\Phi(A)$ is idempotent whenever $A$ is idempotent. A map $\Phi$ is left linear, or shortly linear, if $\Phi(s A+t B)=s \Phi(A)+t \Phi(B)$ for all $s, t \in \mathbb{S}$ and $A, B \in M_{n}(\mathbb{S})$. We say that $A$ and $B$ are orthogonal if $A B=0=B A$. The Schur, i.e., entrywise product of matrices $A$ and $B$ is denoted by $A \circ B$.

We now state the main results of this paper.

Theorem 2.1. Let a semiring $\mathbb{S}$ be $C O M, M C$, and not $A N$. If $n \geq 2$ and $1+1 \neq 0$, then a linear map $\Phi: M_{n}(\mathbb{S}) \rightarrow M_{n}(\mathbb{S})$ preserves idempotents if and only if it satisfies one of the following forms:

$$
\begin{aligned}
s \Phi(A) & =Q A R, \\
s \Phi(A) & =Q A^{\mathrm{tr}} R, \\
\Phi & \equiv 0 .
\end{aligned}
$$

Here, $s \in \mathbb{S}$ is nonzero and matrices $Q, R \in M_{n}(\mathbb{S})$ satisfy $Q R=R Q=s I$.

Remark 2.2. We cannot always assume that $s=1$ (see Counterexample 5.6).

Theorem 2.3. Let a semiring $\mathbb{S}$ be $A C, Z D F$, and $A N$. If $n \geq 2$ and a linear $\operatorname{map} \Phi: M_{n}(\mathbb{S}) \rightarrow M_{n}(\mathbb{S})$ preserves idempotents, then it fits one of the following forms:

$$
\begin{aligned}
& \Phi(A)=P(A \circ X) P^{-1} \\
& \Phi(A)=P\left(A^{\operatorname{tr} \circ X) P^{-1}}\right. \\
& \Phi(A)=\sum_{i=1}^{n} a_{i i} P_{i} \\
& \Phi(A)=P\left(\begin{array}{cc}
a_{11} y_{11} & a_{12} y_{12}+a_{21} z_{21} \\
a_{21} y_{21}+a_{12} z_{12} & a_{22} y_{22}
\end{array}\right) P^{-1} \quad(n=2) .
\end{aligned}
$$

Here, $P$ is a permutation matrix, $X \in M_{n}(\mathbb{S})$ has nonzero entries, $P_{1}, \ldots, P_{n}$ are pairwise orthogonal (possibly zero) idempotents, and $a_{i j}$ denotes the $(i, j)$ entry of the matrix $A$. In (iv'), $y_{i i}^{2}=y_{i i} \neq 0$, and $y_{i j}=0$ or $z_{i j}=0$ for all $i \neq j$.

Remark 2.4. We will see in the proof that entries of the matrix $X$ satisfy $x_{i i}^{2}=x_{i i}$ and $x_{i j} t x_{j k}=t x_{i k}$ for all $i, j, k$ distinct and for all $t \in \mathbb{S}$.

We also remark that the inverse of Theorem 2.3 is not true in general, i.e., not every map (i')-(iv') preserves idempotents. If we strengthen ZDF to MCL or MCR, then we get a stronger result.

Corollary 2.5. Let $\mathbb{S}$ be $A C, M C L$ or $M C R$, and $A N$. If $n \geq 3$ and a linear $\operatorname{map} \Phi: M_{n}(\mathbb{S}) \rightarrow M_{n}(\mathbb{S})$ preserves idempotents, then it fits one of the following 
forms:

$$
\begin{aligned}
& \Phi(A)=Q A Q^{-1}, \\
& \Phi(A)=Q A^{\operatorname{tr}} Q^{-1} \quad(\mathbb{S} \text { is } C O M), \\
& \Phi(A)=\sum_{i=1}^{n} a_{i i} P_{i} .
\end{aligned}
$$

Here, $Q \in M_{n}(\mathbb{S})$ is invertible and such that all entries of $Q$ and $Q^{-1}$ are in $Z(\mathbb{S})$.

We can even drop the AN assumption if we add COM. Note that in this case $\mathrm{MCL}=\mathrm{MC}=\mathrm{MCR}$.

Corollary 2.6. Let a semiring $\mathbb{S}$ be $C O M, M C$, and $A C$. If $n \geq 3$ and $1+1 \neq 0$, then a linear map $\Phi: M_{n}(\mathbb{S}) \rightarrow M_{n}(\mathbb{S})$ preserves idempotents if and only if it satisfies one of the forms (i), (ii), (iii), or it is nonzero and of the form (iii"), but only if every idempotent matrix in $M_{n}(\mathbb{S})$ has all diagonal entries equal 0 or 1.

\section{Proofs}

It is well known that any commutative ring $\mathbb{S}$, which is zero divisor free, can be embedded into the field of fractions, denoted here by $\mathbb{F}(\mathbb{S}$ ) (see e.g. [6, Section 6.2$]$ ). Recall that $\mathbb{F}(\mathbb{S})$ consists of all equivalence classes $[a, b]$ via the equivalence relation

$$
(a, b) \sim(c, d) \Leftrightarrow a d=b c
$$

defined on $\mathbb{S} \times \mathbb{S} \backslash\{0\}$. Addition and multiplication are given by $[a, b]+[c, d]:=$ $[a d+b c, b d]$ and $[a, b] \cdot[c, d]:=[a c, b d]$.

Now, if a semiring $\mathbb{S}$ is COM and MC, then $\mathbb{F}(\mathbb{S})$ has all properties of a field with exception that $(\mathbb{F}(\mathbb{S}),+)$ is only a monoid and not necessarily a group (cf. $[9$, p. 106, Theorem 2.5]). Moreover, the map $\varphi: \mathbb{S} \rightarrow \mathbb{F}(\mathbb{S})$, defined by

$$
\varphi(s):=[s, 1],
$$

is an injective semiring homomorphism.

Lemma 3.1. If a semiring $\mathbb{S}$ is $C O M, M C$, and not $A N$, then $\mathbb{F}(\mathbb{S})$ is a field.

Proof. Since $\mathbb{S}$ is not $\mathrm{AN}$, there exist nonzero $x, y \in \mathbb{S}$ such that $x+y=0$. Therefore, given $[a, b] \in \mathbb{F}(\mathbb{S})$ we can define its additive inverse by $[y a, x b]$. Namely, $[a, b]+[y a, x b]=[x a, x b]+[y a, x b]=[(x+y) a, x b]=0$. Hence, $(\mathbb{F}(\mathbb{S}),+)$ is a group.

If $\mathbb{F}$ is a field, and nonzero idempotent matrices $P_{1}, \ldots, P_{n} \in M_{n}(\mathbb{F})$ are pairwise orthogonal, then there exists an invertible matrix $Q \in M_{n}(\mathbb{F})$ such that $P_{i}=Q E_{i i} Q^{-1}$ for all $i$ (see [4, Lemma 2.2] or [10, p. 62, Exercise 1] for a generalization of this). Similar conclusion holds for semirings, which are COM, $\mathrm{MC}$, and are not $\mathrm{AN}$. 
Lemma 3.2. Let the semiring $\mathbb{S}$ be $C O M, M C$, and not $A N$. If nonzero idempotents $P_{1}, \ldots, P_{n} \in M_{n}(\mathbb{S})$ are pairwise orthogonal, then there exist a nonzero $s \in \mathbb{S}$ and matrices $Q, R \in M_{n}(\mathbb{S})$ with $Q R=R Q=s I$ such that $s P_{i}=Q E_{i i} R$ for all $i$.

Proof. For a given matrix $A \in M_{n}(\mathbb{S})$ let $A^{\varphi}$ be a matrix obtained from $A$ by applying $\varphi$ from (1) entrywise. Clearly, $P_{1}^{\varphi}, \ldots, P_{n}^{\varphi} \in M_{n}(\mathbb{F}(\mathbb{S}))$ are nonzero pairwise orthogonal idempotents. Since $\mathbb{F}(\mathbb{S})$ is a field by Lemma 3.1, there exists an invertible matrix $V \in M_{n}(\mathbb{F}(\mathbb{S}))$ such that $P_{i}^{\varphi}=V E_{i i} V^{-1}$ for all $i$. Hence, $\left(\sum_{i=1}^{n} P_{i}\right)^{\varphi}=\sum_{i=1}^{n} P_{i}^{\varphi}=I=I^{\varphi}$, and consequently

$$
\sum_{i=1}^{n} P_{i}=I
$$

Let $W:=V^{-1}$ and denote fractions at the $(i, j)$-entry of the matrices $V$ and $W$ with $\left[v_{i j}, v_{i j}^{\prime}\right]$ and $\left[w_{i j}, w_{i j}^{\prime}\right]$ respectively. Further, let $\alpha:=\left[\prod_{i, j=1}^{n} v_{i j}^{\prime}, 1\right], \beta:=$ $\left[\prod_{i, j=1}^{n} w_{i j}^{\prime}, 1\right]$, and $\gamma:=\alpha \beta$. Clearly, $\gamma \neq 0$ and

$$
\gamma P_{i}^{\varphi}=(\alpha V) E_{i i}(\beta W) \quad(i=1, \ldots, n) .
$$

Now, $\alpha V=Q^{\varphi}$ and $\beta W=R^{\varphi}$ for some $Q, R \in M_{n}(\mathbb{S})$. Similarly, $\gamma=\varphi(s)$ for nonzero $s:=\left(\prod_{i, j=1}^{n} v_{i j}^{\prime}\right) \cdot\left(\prod_{i, j=1}^{n} w_{i j}^{\prime}\right) \in \mathbb{S}$. Therefore,

$$
s P_{i}=Q E_{i i} R \quad(i=1, \ldots, n) .
$$

By (2) and (3) we deduce that $s I=\sum_{i=1}^{n} s P_{i}=Q R$.

To show that $R Q=s I$, let $t_{i j}$ denote the $(i, j)$-th entry of the matrix $R Q$. Then,

(4) $\quad s^{2} P_{i}=\left(s P_{i}\right)^{2}=Q\left(E_{i i} R Q E_{i i}\right) R=t_{i i} Q E_{i i} R=t_{i i} s P_{i}$.

By assumption, $P_{i} \neq 0$ and $\mathbb{S}$ is MC. Hence, (4) implies that $t_{i i}=s$. If $i \neq j$, then

(5) $0=\left(s P_{i}\right)\left(s P_{j}\right)=Q\left(E_{i i} R Q E_{j j}\right) R=t_{i j} Q E_{i j} R=t_{i j}\left(Q \mathbf{e}_{i}\right)\left(R^{\operatorname{tr}} \mathbf{e}_{j}\right)^{\operatorname{tr}}$,

where $\mathbf{e}_{i}$ and $\mathbf{e}_{j}$ are column-vectors with 1 at the $i$-th, respectively $j$-th, component and zeros elsewhere. If $t_{i j} \neq 0$, then (5) implies that $Q \mathbf{e}_{i}=0$ or $R^{\operatorname{tr}} \mathbf{e}_{j}=0$, because $\mathbb{S}$ is MC. Consequently, $s P_{i}=\left(Q \mathbf{e}_{i}\right)\left(R^{\operatorname{tr}} \mathbf{e}_{i}\right)^{\operatorname{tr}}=0$ or $s P_{j}=\left(Q \mathbf{e}_{j}\right)\left(R^{\operatorname{tr}} \mathbf{e}_{j}\right)^{\operatorname{tr}}=0$, a contradiction. Hence, $t_{i j}=0$ and $R Q=s I$.

For semirings which are ZDF and AN the following analogue holds.

Lemma 3.3. Let a semiring $\mathbb{S}$ be ZDF and $A N$. If nonzero idempotents $P_{1}, \ldots$, $P_{n} \in M_{n}(\mathbb{S})$ are pairwise orthogonal, then there exist a permutation matrix $P$ and nonzero $s_{1}, \ldots, s_{n} \in \mathbb{S}$ such that $s_{i}^{2}=s_{i}$ and $P_{i}=P\left(s_{i} E_{i i}\right) P^{-1}$ for all $i$.

Proof. It suffices to show that, as sets, $\left\{P_{1}, \ldots, P_{n}\right\}=\left\{s_{1} E_{11}, \ldots, s_{n} E_{n n}\right\}$ for some $s_{i}=s_{i}^{2} \neq 0$. Let $p_{j k}^{(i)}$ denote the $(j, k)$-th entry of the matrix $P_{i}$. Since $P_{i} P_{j}=0$ for all $i \neq j$, we deduce that $\sum_{k=1}^{n} p_{g k}^{(i)} p_{k h}^{(j)}=0$ for all $g$ and $h$. Since 
$\mathbb{S}$ is AN, this means that $p_{g k}^{(i)} p_{k h}^{(j)}=0$ for all $k$. Because $\mathbb{S}$ is ZDF, we see that if $p_{g k}^{(i)} \neq 0$, then

(6)

all entries of the matrix $P_{j}$ in the $k$-th row and $g$-th column vanish $(j \neq i)$.

We will now use induction on $n$. Let $n=2$. If $p_{12}^{(1)} \neq 0\left(\right.$ or $\left.p_{21}^{(1)} \neq 0\right)$, then (6) implies that $P_{2}=p_{21}^{(2)} E_{21}$ (or $\left.P_{2}=p_{12}^{(2)} E_{12}\right)$. This is a contradiction since $P_{2}$ is a nonzero idempotent. Hence, $P_{1}$ is a diagonal $2 \times 2$ matrix. By symmetry the same holds for $P_{2}$. Since $\mathbb{S}$ is ZDF and $P_{1}, P_{2}$ are nonzero, we easily deduce that $\left\{P_{1}, P_{2}\right\}=\left\{s_{1} E_{11}, s_{2} E_{22}\right\}$ for some nonzero $s_{1}, s_{2} \in \mathbb{S}$ with $s_{1}^{2}=s_{1}, s_{2}^{2}=s_{2}$. Assume now the conclusion holds for $n=m-1$. Let $n=m$.

If there exist $g_{1}, \ldots, g_{m}$ and $h_{1}, \ldots, h_{m}$ such that $\left\{g_{1}, \ldots, g_{m}\right\}=\{1, \ldots, m\}$ $=\left\{h_{1}, \ldots, h_{m}\right\}$ and $p_{g_{i} h_{i}}^{(i)} \neq 0$ for all $i$, then (6) implies that $P_{i}=p_{g_{i} h_{i}}^{(i)} E_{g_{i} h_{i}}$. Since $P_{i}$ is a nonzero idempotent, we deduce that $g_{i}=h_{i}$ and $s_{i}:=p_{g_{i} h_{i}}^{(i)}=$ $\left(p_{g_{i} h_{i}}^{(i)}\right)^{2}$. Hence, $\left\{P_{1}, \ldots, P_{m}\right\}=\left\{s_{1} E_{11}, \ldots, s_{m} E_{m m}\right\}$.

Assume erroneously that such $g_{1}, \ldots, g_{m}$ and $h_{1}, \ldots, h_{m}$ do not exist. Then all matrices $P_{1}, \ldots, P_{m}$ have some fixed row or column zero. Say that $r$ th row is such (the proof is symmetrical if $r$-th column is zero). Then, all matrices $P_{i}$ must have some nonzero entry outside of $r$-th column and row. Otherwise we would deduce $P_{i}=P_{i}^{2}=0$ by a straightforward calculation. Hence, the $(m-1) \times(m-1)$ matrices $\tilde{P}_{i}$, which are obtained from matrices $P_{i}$ by deleting $r$-th column and row, are nonzero. Since matrices $P_{i}$ have $r$-th row zero it follows that $\tilde{P}_{i}^{2}=\tilde{P}_{i}$ for all $i=1, \ldots, m$ and $\tilde{P}_{i} \tilde{P}_{j}=0$ for all $i \neq j$. By induction, $\left\{\tilde{P}_{1}, \tilde{P}_{2}, \ldots, \tilde{P}_{m-1}\right\}=\left\{s_{1} E_{11}, s_{2} E_{22}, \ldots, s_{m-1} E_{m-1, m-1}\right\}$ for some $s_{i}=s_{i}^{2} \neq 0$. In the same way we infer that $\left\{\tilde{P}_{2}, \tilde{P}_{3}, \ldots, \tilde{P}_{m}\right\}=$ $\left\{t_{1} E_{11}, t_{2} E_{22}, \ldots, t_{m-1} E_{m-1, m-1}\right\}$ for some $t_{i}=t_{i}^{2} \neq 0$. Therefore, there exist $i, j \in\{1, \ldots, m-1\}$ such that $\tilde{P}_{m}=t_{i} E_{i i}$ and $\tilde{P}_{j}=s_{i} E_{i i}$. Consequently, $\tilde{P}_{j} \tilde{P}_{m}=s_{i} t_{i} E_{i i} \neq 0$, a contradiction.

Lemma 3.4. Assume a semiring $\mathbb{S}$ has at least three elements and (a) is COM and $M C$ or (b) is $A C$ and $A N$. Suppose $n \geq 2$ and $\Phi: M_{n}(\mathbb{S}) \rightarrow M_{n}(\mathbb{S})$ is a linear map which preserves idempotents. If $\Phi\left(E_{i i}\right)=0$ for some $i$, then $\Phi\left(E_{j k}\right)=0$ for all $j \neq k$.

Proof. Let $\Phi\left(E_{i i}\right)=0$ and choose $j \neq i$ arbitrarily. Since $E_{i i}+s E_{i j}$ is an idempotent for all $s \in \mathbb{S}$, the same holds for $\Phi\left(E_{i i}+s E_{i j}\right)=s \Phi\left(E_{i j}\right)$. Hence,

$$
s \Phi\left(E_{i j}\right) s \Phi\left(E_{i j}\right)=s \Phi\left(E_{i j}\right) \quad(s \in \mathbb{S}) .
$$

Now, if $\mathbb{S}$ is COM and MC, then (7) implies that

$$
s \Phi\left(E_{i j}\right)^{2}=\Phi\left(E_{i j}\right)=t \Phi\left(E_{i j}\right)^{2} \quad(s, t \neq 0) .
$$

Since $|\mathbb{S}| \geq 3$, we can choose such $s$ and $t$ distinct. Therefore, $\Phi\left(E_{i j}\right)=0$.

Otherwise, if $\mathbb{S}$ is $\mathrm{AC}$ and $\mathrm{AN}$, choose $s=1+1=: 2$ and $s=1$ in (7) to deduce $2 \Phi\left(E_{i j}\right)=4 \Phi\left(E_{i j}\right)^{2}=2 \Phi\left(E_{i j}\right)^{2}+2 \Phi\left(E_{i j}\right)^{2}=2 \Phi\left(E_{i j}\right)+2 \Phi\left(E_{i j}\right)$ 
(note that $2 \in Z(\mathbb{S}))$. Now, AC implies that $0=2 \Phi\left(E_{i j}\right)=\Phi\left(E_{i j}\right)+\Phi\left(E_{i j}\right)$. Consequently, by AN,

$$
\Phi\left(E_{i j}\right)=0 \quad(j \neq i) .
$$

We deduce that

$$
\Phi\left(E_{j i}\right)=0 \quad(j \neq i)
$$

in the same way as (9). This ends the proof if $n=2$. Otherwise, choose $j$ and $k$ such that $i, j, k$ are all distinct. Since $E_{i i}+E_{j i}+s E_{i k}+s E_{j k}$ is an idempotent for all $s$, the same is true for $\Phi\left(E_{i i}+E_{j i}+s E_{i k}+s E_{j k}\right)=s \Phi\left(E_{j k}\right)$. We repeat the procedure above to deduce that $\Phi\left(E_{j k}\right)=0$.

Proof of Theorem 2.1. We first prove the "if part". If $\Phi$ satisfies (i) and $A^{2}=$ $A$, then $s^{2} \Phi(A)^{2}=(s \Phi(A))^{2}=Q A R Q A R=s Q A^{2} R=s Q A R=s^{2} \Phi(A)$. Since $\mathbb{S}$ is MC, we deduce that $\Phi(A)^{2}=\Phi(A)$, i.e., $\Phi$ preserves idempotents. We proceed in the same way if $\Phi$ satisfies (ii). The zero map (iii) preserves idempotents as well.

It remains to prove the "only if part". Choose arbitrary distinct $i$ and $j$. Matrices $\Phi\left(E_{i i}+E_{j j}\right), \Phi\left(E_{i i}\right)$, and $\Phi\left(E_{j j}\right)$ are idempotents, i.e.,

$$
\Phi\left(E_{i i}\right)+\Phi\left(E_{j j}\right)=\Phi\left(E_{i i}\right)+\Phi\left(E_{j j}\right)+\Phi\left(E_{i i}\right) \Phi\left(E_{j j}\right)+\Phi\left(E_{j j}\right) \Phi\left(E_{i i}\right) .
$$

Note that $\mathbb{S}$ is AC by Lemma 3.1. Hence, the above implies that $\Phi\left(E_{i i}\right) \Phi\left(E_{j j}\right)+$ $\Phi\left(E_{j j}\right) \Phi\left(E_{i i}\right)=0$. Multiply this equation with $\Phi\left(E_{i i}\right)$ from the left, i.e.,

$$
\Phi\left(E_{i i}\right) \Phi\left(E_{j j}\right)+\Phi\left(E_{i i}\right) \Phi\left(E_{j j}\right) \Phi\left(E_{i i}\right)=0,
$$

and equation (10) with $\Phi\left(E_{i i}\right)$ from the right. We deduce that

$$
2 \Phi\left(E_{i i}\right) \Phi\left(E_{j j}\right) \Phi\left(E_{i i}\right)=0 \text {. }
$$

Since $\mathbb{S}$ is MC with $2 \neq 0$, it follows that $\Phi\left(E_{i i}\right) \Phi\left(E_{j j}\right) \Phi\left(E_{i i}\right)=0$. We infer that

$$
\Phi\left(E_{i i}\right) \Phi\left(E_{j j}\right)=0 \quad(i \neq j)
$$

from (10). Note that $2=1+1 \neq 1$ by AC. Hence, $|\mathbb{S}| \geq 3$.

Assume first that $\Phi\left(E_{i i}\right)=0$ for some $i$. Then, $\Phi(A)=\sum_{k=1}^{n} a_{k k} \Phi\left(E_{k k}\right)$ by Lemma 3.4. Here, $a_{k k}$ is the $(k, k)$-entry of the matrix $A$. Since $\mathbb{S}$ is not AN, there exist nonzero $x, y \in \mathbb{S}$ such that $x+y=0$. We may assume that $x \neq 1$. Otherwise replace $x$ with $2 x$ and $y$ with $2 y$. Now, let $j \neq i$ be arbitrary. Since

$$
B:=(y+1) E_{i i}+x(y+1) E_{i j}+E_{j i}+x E_{j j} \text { and } E_{j j}
$$

are idempotents it follows that $x^{2} \Phi\left(E_{j j}\right)=x \Phi\left(E_{j j}\right)$. Note that $x^{2} \neq x$, since $x \neq 0,1$ and $\mathbb{S}$ is MC. Consequently, $\Phi\left(E_{j j}\right)=0$. Since $j$ is arbitrary, it follows that $\Phi \equiv 0$, i.e., $\Phi$ is of the form (iii).

If $\Phi\left(E_{i i}\right) \neq 0$ for all $i$, then, by Lemma 3.2 and (11), there exist matrices $Q, R$ and nonzero $s \in \mathbb{S}$ such that

$$
s \Phi\left(E_{i i}\right)=Q E_{i i} R \quad(i=1, \ldots, n)
$$


and $Q R=R Q=s I$. If $i \neq j$, then $\Phi\left(E_{i i}+t E_{i j}\right)$ is an idempotent for any $t$. Hence, $\Phi\left(E_{i i}\right)+t \Phi\left(E_{i j}\right)=\Phi\left(E_{i i}\right)+t^{2} \Phi\left(E_{i j}\right)^{2}+t \Phi\left(E_{i i}\right) \Phi\left(E_{i j}\right)+t \Phi\left(E_{i j}\right) \Phi\left(E_{i i}\right)$. By $\mathrm{AC}$,

$$
t \Phi\left(E_{i j}\right)=t^{2} \Phi\left(E_{i j}\right)^{2}+t \Phi\left(E_{i i}\right) \Phi\left(E_{i j}\right)+t \Phi\left(E_{i j}\right) \Phi\left(E_{i i}\right) .
$$

Combine equation $((14), t=2)$ together with equation $((14), t=1)$ multiplied by 2 . We deduce that $2 \Phi\left(E_{i j}\right)^{2}=0$ by AC. Hence, $\Phi\left(E_{i j}\right)^{2}=0$, i.e., $s \Phi\left(E_{i j}\right)=$ $s \Phi\left(E_{i i}\right) \Phi\left(E_{i j}\right)+\Phi\left(E_{i j}\right) s \Phi\left(E_{i i}\right)$. Consequently, we infer from (13) that

$$
R \Phi\left(E_{i j}\right) Q=E_{i i} R \Phi\left(E_{i j}\right) Q+R \Phi\left(E_{i j}\right) Q E_{i i} .
$$

In the same way we deduce that

$$
R \Phi\left(E_{i j}\right) Q=E_{j j} R \Phi\left(E_{i j}\right) Q+R \Phi\left(E_{i j}\right) Q E_{j j} .
$$

Equations (15) and (16) imply that $R \Phi\left(E_{i j}\right) Q=x_{i j} E_{i j}+y_{i j} E_{j i}$, i.e.,

$$
s^{2} \Phi\left(E_{i j}\right)=Q\left(x_{i j} E_{i j}+y_{i j} E_{j i}\right) R
$$

for some $x_{i j}, y_{i j} \in \mathbb{S}$. Since $\Phi\left(E_{i j}\right)^{2}=0$, we deduce that $x_{i j} y_{i j}=0$, i.e., $x_{i j}=0$ or $y_{i j}=0$. From (13), (17), and equation $\left(s^{2} \Phi(B)\right)^{2}=s^{2}\left(s^{2} \Phi(B)\right)$, where $B$ is defined in (12), we infer that $(y+1)\left(x_{i j} x_{j i}+y_{i j} y_{j i}\right)+s^{2} x=s^{2}$. Add $s^{2} y$ to both sides of this equation. Since $x \neq 1$, i.e., $y+1 \neq 0$, we deduce that

$$
x_{i j} x_{j i}+y_{i j} y_{j i}=s^{2} \text {. }
$$

Hence, exactly one element in $\left\{x_{i j}, y_{i j}\right\}$ is nonzero. Moreover, $x_{i j} \neq 0 \Leftrightarrow x_{j i} \neq$ 0 .

Now, for arbitrary $i$, the equation $\left(s^{2} \Phi\left(\sum_{k=1}^{n} E_{i k}\right)\right)^{2}=s^{2}\left(s^{2} \Phi\left(\sum_{k=1}^{n} E_{i k}\right)\right)$, together with (13) and (17), shows that either $x_{i k}=0$ and $y_{i k} \neq 0$ for all $k \neq i$ or conversely $x_{i k} \neq 0$ and $y_{i k}=0$ for all $k \neq i$. Consequently, it follows that

$$
s^{2} \Phi\left(E_{i j}\right)=Q x_{i j} E_{i j} R \quad(\forall i \forall j, i \neq j)
$$

or

$$
s^{2} \Phi\left(E_{i j}\right)=Q y_{i j} E_{j i} R \quad(\forall i \forall j, i \neq j) .
$$

We may assume that (19) is correct. Otherwise consider the map $\Psi(A):=$ $\Phi(A)^{\operatorname{tr}}$.

Equation (18) transforms now into $x_{i j} x_{j i}=s^{2}$. If $n \geq 3$, then $C:=E_{i i}+$ $E_{i k}+E_{j i}+E_{j k}$ is idempotent for arbitrary distinct $i, j, k$. Hence, the equation

$$
x_{j i} x_{i k}=s x_{j k}
$$

follows from $\left(s^{2} \Phi(C)\right)^{2}=s^{2}\left(s^{2} \Phi(C)\right)$. If we define $x_{i i}:=s$ for all $i$, then $(21)$ holds for arbitrary $i, j, k$ (they are allowed to be equal). By (19), $s \Phi\left(E_{i j}\right) Q=$ $Q x_{i j} E_{i j}$ and $s R \Phi\left(E_{i j}\right)=x_{i j} E_{i j} R$. Hence, the $i$-th column of the matrix $Q x_{i j}$ and the $j$-th row of the matrix $x_{i j} R$ are divisible by $s$, i.e., every their entry is of the form st for some $t$ (this is obvious when $i=j$ ). Consequently, $Q \operatorname{diag}\left(x_{11}, x_{21}, \ldots, x_{n 1}\right)=s \tilde{Q}$ and $\operatorname{diag}\left(x_{11}, x_{12}, \ldots, x_{1 n}\right) R=s \tilde{R}$ for some matrices $\tilde{Q}$ and $\tilde{R}$. It follows from (21) that $s \tilde{Q} s \tilde{R}=s^{3} I=s \tilde{R} s \tilde{Q}$, i.e., $\tilde{Q} \tilde{R}=\tilde{R} \tilde{Q}=s I$. Again, by (21), $s \tilde{Q} E_{i j} s \tilde{R}=Q s x_{i j} E_{i j} R=s^{3} \Phi\left(E_{i j}\right)$, i.e., 
$s \Phi\left(E_{i j}\right)=\tilde{Q} E_{i j} \tilde{R}$ for all $i$ and $j$. Therefore, $\Phi$ satisfies (i). Clearly, if (20) is correct instead of (19), then $\Phi$ is of the form (ii).

A big part of the proof of Theorem 2.3 is almost identical as its counterpart in Theorem 2.1. Therefore we only sketch the differences.

Sketch of the proof of Theorem 2.3. Note that the equation $2 t=0$ still implies $t=0$, since $\mathbb{S}$ is AN. Hence, we deduce (11) as in the proof above. Since $\mathbb{S}$ is also $\mathrm{AC}$, we see that $1 \neq 1+1 \neq 0$, i.e., $|\mathbb{S}| \geq 3$. Therefore, if $\Phi\left(E_{i i}\right)=0$ for some $i$, we infer that $\Phi(A)=\sum_{k=1}^{n} a_{k k} \Phi\left(E_{k k}\right)$ from Lemma 3.4. Hence, $\Phi$ is of the form (iii') for $P_{k}=\Phi\left(E_{k k}\right)$. If $\Phi\left(E_{i i}\right) \neq 0$ for all $i$, then, by Lemma 3.3, there exist a permutation matrix $P$ and $x_{11}, \ldots, x_{n n} \in \mathbb{S} \backslash\{0\}$ such that

$$
\Phi\left(E_{i i}\right)=P\left(x_{i i} E_{i i}\right) P^{-1}
$$

and $x_{i i}^{2}=x_{i i}$ for all $i$. Since $\Phi\left(E_{i i}+E_{i j}\right)$ and $\Phi\left(E_{i i}+2 E_{i j}\right)$ are idempotents for $i \neq j$, and since $2 \in Z(\mathbb{S})$, we deduce that

$$
\Phi\left(E_{i j}\right)=P\left(x_{i j} E_{i j}+y_{i j} E_{j i}\right) P^{-1}
$$

for some $x_{i j}, y_{i j} \in \mathbb{S}$, similarly as in the previous proof. Again, $x_{i j}=0$ or $y_{i j}=0$. If $n=2$, then $\Phi$ is of the form (iv') by (22) and (23). Let $n \geq 3$. If $\Phi\left(E_{i j}\right)=0$ for all $i$ and $j$ distinct, then $\Phi$ is of the form (iii'). Assume now that $\Phi\left(E_{i j}\right) \neq 0$ for some $i \neq j$. We may say that $x_{i j} \neq 0$ and $y_{i j}=0$ (the proof is symmetrical if $x_{i j}=0$ and $\left.y_{i j} \neq 0\right)$. Since matrices $\Phi\left(\sum_{k=1}^{n} E_{i k}\right)$ and $\Phi\left(\sum_{k=1}^{n} E_{k j}\right)$ are idempotents, and $x_{i j} \neq 0$, we deduce that $y_{i k}=0$ for all $k \neq i$ and $y_{k j}=0$ for all $k \neq j$. Choose $k \neq i, j$ and $t \in \mathbb{S}$ arbitrarily. Matrix $\Phi\left(t E_{i j}+E_{i k}+t E_{k j}+E_{k k}\right)$ is idempotent. Hence,

$$
x_{i k} t x_{k j}=t x_{i j} .
$$

In particular, $x_{i k}$ and $x_{k j}$ are nonzero. Since $n \geq 3$ and $k$ is arbitrary, we can repeat the procedure above and deduce that

$$
\Phi\left(E_{i j}\right)=P\left(x_{i j} E_{i j}\right) P^{-1} \neq 0
$$

for all $i$ and $j$. Moreover, (24) holds for arbitrary distinct $i, j, k$. Hence, $\Phi$ is of the form (i') for $X:=\left[x_{i j}\right]$. If we would assume that $x_{i j}=0$ and $y_{i j} \neq 0$, then $\Phi$ would be of the form (ii') for $X:=\left[y_{i j}\right]^{\text {tr }}$, where $y_{i i}:=x_{i i}$.

Proof of Corollary 2.5. If $\Phi$ is not of the form (iii"), then it is of the form (i') or (ii') by Theorem 2.3. By Remark 2.4, $x_{i i}^{2}=x_{i i} \neq 0$, i.e., $x_{i i}=1$ since $\mathbb{S}$ is MCL or MCR. Moreover, $x_{k j} x_{j i} x_{i j}=x_{k i} x_{i j}=x_{k j} \neq 0$. Similarly, $x_{j i} x_{i j} x_{j k}=$ $x_{j k} \neq 0$. Hence, by MCL/MCR, $x_{j i} x_{i j}=1$ for all $i \neq j$. Now, Remark 2.4 implies that $x_{i j} t=x_{i j} t x_{j k} x_{k j}=t x_{i k} x_{k j}=t x_{i j}$, so the matrix $X$ has all entries in $Z(\mathbb{S})$. Let $V$ be the diagonal matrix $\operatorname{diag}\left(x_{11}, x_{21}, \ldots, x_{n 1}\right)$. Then, $V^{-1}=$ $\operatorname{diag}\left(x_{11}, x_{12}, \ldots, x_{1 n}\right)$. Moreover, $A \circ X=V A V^{-1}$ and $A^{\operatorname{tr}} \circ X=V A^{\operatorname{tr}} V^{-1}$ for all $A$. Hence, the form (i') gives (i") and the form (ii') gives (ii"). If $\Phi$ is of the form (ii"), then $s t=t s$ for any $s, t \in \mathbb{S}$, since $\Phi\left(E_{11}+s E_{21}+t E_{13}+s t E_{23}\right)$ is idempotent. Hence, $\mathbb{S}$ is COM. 
Proof of Corollary 2.6. The "if" part is obvious. We will prove the "only if" part. If $\mathbb{S}$ is not $A N$, then it satisfies the assumptions of Theorem 2.1 and $\Phi$ fits one of the forms (i)-(iii). If $\mathbb{S}$ is $\mathrm{AN}$, then it satisfies the assumptions of Corollary 2.5 and $\Phi$ fits one of the forms (i")-(iii"). Clearly, the forms (i") and (ii") are a special type of (i) and (ii) respectively. Suppose now that $\Phi$ preserves idempotents and is of the form (iii"). If it is not the zero map (iii), then there exists $i$ such that $P_{i} \neq 0$. Choose an idempotent $A=\left[a_{j k}\right] \in M_{n}(\mathbb{S})$ and $j \in\{1, \ldots, n\}$ arbitrarily. If a permutation matrix $P$ is such that the $(i, i)$ th entry of the matrix $P A P^{-1}$ equals $a_{j j}$, then the equation $\Phi\left(P A P^{-1}\right)^{2} P_{i}=$ $\Phi\left(P A P^{-1}\right) P_{i}$ implies that $a_{j j}^{2} P_{i}=a_{j j} P_{i}$. Since $P_{i} \neq 0$, it follows that $a_{j j}^{2}=$ $a_{j j}$, i.e., $a_{j j} \in\{0,1\}$.

\section{Direct products of semirings}

If $\left\{\mathbb{S}_{\lambda} \mid \lambda \in \Lambda\right\}$ is a family of semirings, then the direct product $\mathbb{S}=\times_{\lambda \in \Lambda} \mathbb{S}_{\lambda}$ is a semiring with the operations of addition and multiplication defined componentwise. Given $s \in \mathbb{S}$ let $s_{\lambda} \in \mathbb{S}_{\lambda}$ be its $\lambda$-th component. Similarly, for $A=\left[a_{i j}\right] \in M_{n}(\mathbb{S})$ let $A_{\lambda}$ denote the matrix $\left[\left(a_{i j}\right)_{\lambda}\right] \in M_{n}\left(\mathbb{S}_{\lambda}\right)$. Note that $(A+B)_{\lambda}=A_{\lambda}+B_{\lambda}$ and

$$
(A B)_{\lambda}=A_{\lambda} B_{\lambda}
$$

for arbitrary $A, B \in M_{n}(\mathbb{S})$.

Lemma 4.1. If $\Phi: M_{n}(\mathbb{S}) \rightarrow M_{n}(\mathbb{S})$ is a linear map, then, for each $\lambda \in \Lambda$, there exists a unique linear map $\Phi_{\lambda}: M_{n}\left(\mathbb{S}_{\lambda}\right) \rightarrow M_{n}\left(\mathbb{S}_{\lambda}\right)$ such that $\Phi(A)_{\lambda}=$ $\Phi_{\lambda}\left(A_{\lambda}\right)$ for all $A \in M_{n}(\mathbb{S})$.

Proof. For any $B \in M_{n}\left(\mathbb{S}_{\lambda}\right)$ define $\Phi_{\lambda}(B):=\Phi(C)_{\lambda}$, where $C \in M_{n}(\mathbb{S})$ is such that $C_{\lambda}=B$ and $C_{\mu}=0$ for $\mu \neq \lambda$. Let $s \in \mathbb{S}$ satisfy $s_{\lambda}=1$ and $s_{\mu}=0$ for $\mu \neq \lambda$. Then, $\Phi(A)_{\lambda}=(s \Phi(A))_{\lambda}=\Phi(s A)_{\lambda}=\Phi_{\lambda}\left(A_{\lambda}\right)$. Clearly, $\Phi_{\lambda}$ is linear and unique.

We infer from (25) that $A$ is idempotent if and only if $A_{\lambda}$ is idempotent for all $\lambda$. Hence, we have the following lemma.

Lemma 4.2. A map $\Phi: M_{n}(\mathbb{S}) \rightarrow M_{n}(\mathbb{S})$ is linear and preserves idempotents if and only if all maps $\Phi_{\lambda}$ are linear and preserve idempotents.

Recall that an element $s$ of a semiring is called multiplicatively cancellable if each of the equations $s t=s u$ and $t s=u s$ implies $t=u$ for any $t$ and $u$.

Theorem 4.3. Let $\mathbb{S}_{\lambda}$ satisfy the assumptions of Theorem 2.1 for all $\lambda \in \Lambda$. If $n \geq 2$, then a linear map $\Phi: M_{n}(\mathbb{S}) \rightarrow M_{n}(\mathbb{S})$ preserves idempotents if and only if there exist a multiplicatively cancellable $s \in \mathbb{S}$, matrices $Q, R \in M_{n}(\mathbb{S})$ with $Q R=R Q=s I$, and orthogonal idempotents $f_{1}, f_{2} \in \mathbb{S}$ such that $\Phi$ satisfies the form

$$
s \Phi(A)=Q\left(f_{1} A+f_{2} A^{\mathrm{tr}}\right) R .
$$


Proof. The "if" part is proved similarly as in Theorem 2.1. To prove the "only if" part assume that $\Phi$ is linear and preserves idempotents. By Lemma 4.2 the same holds for the maps $\Phi_{\lambda}$. By Theorem 2.1 there exist matrices $Q, R \in M_{n}(\mathbb{S})$ and $s \in \mathbb{S}$ such that for any $\lambda$ the map $\Phi_{\lambda}$ fits the form

$s_{\lambda} \Phi_{\lambda}(B)=Q_{\lambda} B R_{\lambda}$, or $s_{\lambda} \Phi_{\lambda}(B)=Q_{\lambda} B^{\operatorname{tr}} R_{\lambda}$, or $s_{\lambda} \Phi_{\lambda}(B)=0=Q_{\lambda} \cdot 0 \cdot R_{\lambda}$, where $s_{\lambda} \neq 0$, i.e., $s$ is multiplicatively cancellable. Moreover, $Q R=R Q=s I$. Denote with $\Lambda_{1}, \Lambda_{2}, \Lambda_{3}$ the sets of all $\lambda$ for which $\Phi_{\lambda}$ is of the first, second, and third form respectively. Let the $\lambda$-th component of $f_{i} \in \mathbb{S}$ be 1 if $\lambda \in \Lambda_{i}$ and 0 otherwise. Then, $s \Phi(A)=Q\left(f_{1} A+f_{2} A^{\text {tr }}+f_{3} \cdot 0\right) R=Q\left(f_{1} A+f_{2} A^{\text {tr }}\right) R$ for all $A$. Clearly, for idempotents $f_{1}$ and $f_{2}, f_{1} f_{2}=0=f_{2} f_{1}$ holds.

An interesting example of a direct product is $\mathbb{Z}_{m}$, the semiring of integers modulo $m$, where $m=p_{1} p_{2} \cdots p_{k}$ is a product of distinct odd primes. In fact, the map $\varphi: \mathbb{Z}_{m} \rightarrow \times_{\lambda=1}^{k} \mathbb{Z}_{p_{\lambda}}$, defined by $\varphi(s)_{\lambda}:=s$, is a semiring isomorphism (surjectiveness is an immediate consequence of the Chinese remainder theorem). Since all $\mathbb{Z}_{p_{\lambda}}$ are fields, we see from the proof above, that in this case $s=1$ and $R=Q^{-1}$. Moreover, $\Phi(A)=e Q\left(f A+(1-f) A^{\text {tr }}\right) Q^{-1}$ for idempotents $e:=f_{1}+f_{2}$ and $f:=f_{1}+f_{3}$.

Though written in a slightly different way, the approach of viewing a semiring as a direct product of "nicer", i.e., MC semirings was used in [13] and [11, Theorem 3.2], where bijective linear preservers of idempotents and linear strong preservers of idempotents on matrices over general finite Boolean algebra were classified by reducing the problem to the analogue for binary Boolean algebra. Recall that a finite Boolean algebra is isomorphic to a direct product of binary Boolean algebras.

Remark 4.4. A similar result to Theorem 4.3 can be proved if some $\mathbb{S}_{\lambda}$ fits the assumptions of Theorem 2.3 instead of Theorem 2.1.

\section{Examples and counterexamples}

Firstly we list some interesting examples of semirings satisfying the assumptions of Theorem 2.1 and Theorem 2.3.

Example 5.1. Any commutative ring with 1, which is zero divisor free and of characteristic not 2 fits the assumptions of Theorem 2.1. However, there exist semirings which are COM, MC, are not AN, and are not rings. An example of such is the semiring of all real polynomials $p$, where the last coefficient, i.e., $p(0)$ is nonnegative. Addition and multiplication are the usual ones.

Example 5.2. Two important semirings which satisfy the assumptions of Theorem 2.3 and Corollary 2.5 are $\mathbb{Z}_{+}$, the semiring of all nonnegative integers, and $\mathbb{R}_{+}$, the semiring of all nonnegative reals, with operations defined as usual. However, there are many more semirings which are AC, ZDF, and AN. In fact, for arbitrary semiring $\mathbb{S}$ there exists a semiring $\mathbb{S}^{\prime}$ which is $\mathrm{AC}, \mathrm{ZDF}$, and $\mathrm{AN}$, 
and a surjective semiring homomorphism $\varphi: \mathbb{S}^{\prime} \rightarrow \mathbb{S}$ (see the construction in $[8$, Proposition 8.33]).

Example 5.3. Let $\mathbb{S}$ be a semiring, $\Sigma$ a nonempty set, and $\Sigma^{*}$ the free monoid defined by $\Sigma$, i.e., $\Sigma^{*}$ consist of all finite words with letters from $\Sigma$, where the operation is just the concatenation of words. The set $\mathbb{S}\langle\langle\Sigma\rangle\rangle$ of all functions $f: \Sigma^{*} \rightarrow \mathbb{S}$, equipped with componentwise addition and multiplication defined by $(f g)(w)=\sum\left\{f\left(w^{\prime}\right) g\left(w^{\prime \prime}\right) \mid w^{\prime} w^{\prime \prime}=w\right\}$, is known as the semiring of formal power series in $\Sigma$ over $\mathbb{S}$. This semiring is a frequently used as a tool in the theory of languages and automata (see [8, pp. 31-33] for some details). It can be proved that if $\mathbb{S}$ satisfies the assumptions of Theorem 2.3 , then so does $\mathbb{S}\langle\langle\Sigma\rangle\rangle$. The analogue holds also for Corollary 2.5. We leave the proofs to the reader.

Note that the map $\Phi$ in Theorem 2.3 can in fact be of the form (iii') or (iv'). Example 5.4. If $\mathbb{S}=\mathbb{Z}_{+}$with usual operations, then the maps (iii') and (iv') always preserve idempotents.

Below we show that, except for COM in Theorem 2.1, neither assumption on the semiring in Theorem 2.1 and Theorem 2.3 can be omitted.

Counterexamples 5.5. (a) We cannot drop MC in Theorem 2.1. Consider the semiring $\mathbb{S}=\times_{\lambda \in \Lambda} \mathbb{S}_{\lambda}$ from Theorem 4.3, where $|\Lambda| \geq 2$, and the map $\Phi(A)=e A$ for $e^{2}=e \notin\{0,1\}$. This semiring satisfies all other assumptions of Theorem 2.1, while $\Phi$ preserves idempotents and is not of the forms (i) and (ii), since $\Phi(I) \neq I$.

(b) We cannot drop the assumption that $\mathbb{S}$ is not AN in Theorem 2.1. Let $\mathbb{S}$ be a binary Boolean algebra, i.e., $\mathbb{S}=\{0,1\}$, where $1+1=1$. Then, $\mathbb{S}$ satisfies all other assumptions of Theorem 2.1. Let $J$ be the matrix with all entries equal 1. The map, defined by $\Phi(0)=0$ and $\Phi(A)=J$ for $A \neq 0$, is linear and preserves idempotents. It is not of the forms (i) and (ii), since its image equals $\{0, J\}=\left\{0, \Phi\left(E_{12}\right)\right\}$.

(c) We cannot drop the assumption that $1+1 \neq 0$ in Theorem 2.1. Any field of characteristic 2 is a semiring that satisfies all other assumptions of Theorem 2.1. The linear map $\Phi(A)=\left(\sum_{i=1}^{n} a_{i i}\right) I$ preserves idempotents. It is not of the forms (i) and (ii), since $\Phi\left(E_{i i}\right)=\Phi\left(E_{j j}\right)$ for all $i$ and $j$. For some other examples see $[1,12]$.

(d) We cannot drop AC in Theorem 2.3. See counterexample in item (b).

(e) We cannot drop ZDF in Theorem 2.3. The semiring $\mathbb{Z}_{+} \times \mathbb{Z}_{+}$with componentwise addition and multiplication satisfies all other assumptions of Theorem 2.3. The linear map $\Phi\left(\left[\left(a_{i j}, b_{i j}\right)\right]\right)=\left[\left(a_{i j}, b_{j i}\right)\right]$ preserves idempotents by Lemma 4.2. It is not of the forms (i')-(iv') since the matrix $\Phi\left(E_{12}\right)$ has two nonzero entries.

(f) We cannot drop AN in Theorem 2.3. See counterexample in item (c).

At the end we show that the conclusions of Theorem 2.1 and Theorem 2.3 are the best possible. 
Counterexample 5.6. In Theorem 2.1 we cannot always assume that $s=1$. If $\mathbb{Z}$ is the set of all integers, then the subset $\mathbb{S}=\{a+i b \sqrt{5} \mid a, b \in \mathbb{Z}\}$ of complex numbers is a semiring for usual addition and multiplication, and satisfies all assumptions of Theorem 2.1. Let

$$
Q=\left(\begin{array}{cc}
3, & 1-i \sqrt{5} \\
-1+i \sqrt{5}, & 2+i \sqrt{5}
\end{array}\right) \quad \text { and } \quad R=\left(\begin{array}{cc}
3, & 1+i \sqrt{5} \\
-1-i \sqrt{5}, & 2-i \sqrt{5}
\end{array}\right)
$$

Then, $Q R=R Q=3 I$ and the map, defined by $3 \Phi(A)=Q A R$, maps $M_{2}(\mathbb{S})$ into $M_{2}(\mathbb{S})$ and preserves idempotents. If there exists an invertible $T=\left[t_{i j}\right] \in$ $M_{2}(\mathbb{S})$ such that $\Phi(A)=T A T^{-1}$ or $\Phi(A)=T A^{\operatorname{tr}} T^{-1}$, then the equation $\Phi\left(E_{11}\right) T=T E_{11}$ implies that $2 t_{11}+(1+i \sqrt{5}) t_{21}=0$ and $3 t_{12}+(1+i \sqrt{5}) t_{22}=$ 0 . Since $T$ is invertible, all entries $t_{i j}$ are nonzero. By equation $\Phi\left(E_{11}\right)=$ $T E_{11} T^{-1}$ we see that the entries of the matrix $T^{-1}$ at positions $(1,1)$ and $(1,2)$ equal $(-1+i \sqrt{5}) / t_{21}$ and $-2 / t_{21}$ respectively. It is now easy to check that at least one of these two numbers or the number $t_{11}$ is not an element of $\mathbb{S}$, for any $t_{21} \in \mathbb{S}$. Hence, such $T$ does not exist.

The difference between Theorem 2.3 and Corollary 2.5 is pointed out below.

Counterexample 5.7. Let $\mathbb{S}=\mathbb{Z}_{+} \times \mathbb{Z}_{+}$with componentwise addition and multiplication given by $(a, b) \cdot(c, d):=(a c, a d+b c+b d)$. This semiring fits Theorem 2.3 but not Corollary 2.5. In fact, it is not MCL/MCR since $(0,1)$. $(1,0)=(0,1) \cdot(0,1)=(1,0) \cdot(0,1)$. Consider the linear map $\Phi: M_{n}(\mathbb{S}) \rightarrow$ $M_{n}(\mathbb{S})$ defined by $\Phi(A)=(0,1) \cdot A$. It preserves idempotents and it is not of the forms (i")-(iii") since $\Phi(I) \neq I$ and $\Phi\left(E_{12}\right) \neq 0$. Less trivial example of a such map is $\Psi(A)=A \circ X$, where all nondiagonal entries of the matrix $X$ equal $(0,1)$, while all diagonal entries equal $(1,0)$. This map is not of the forms (i")-(iii”), since $\Psi\left(E_{12}\right) \Psi\left(E_{21}\right) \notin\left\{\Psi\left(E_{11}\right), \Psi\left(E_{22}\right), 0\right\}$. The proof that $\Psi$ preserves idempotents is left to the reader.

Acknowledgement. The author would like to thank Bojan Kuzma for his kindest suggestions and the referee for his comments.

\section{References}

[1] L. B. Beasley and N. J. Pullman, Linear operators preserving idempotent matrices over fields, Linear Algebra Appl. 146 (1991), 7-20.

[2] _ Linear operators strongly preserving idempotent matrices over semirings, Linear Algebra Appl. 160 (1992), 217-229.

[3] C.-G. Cao and X. Zhang, Linear preservers between matrix modules over connected commutative rings, Linear Algebra Appl. 397 (2005), 355-366.

[4] J.-T. Chan, C.-K. Li, and N.-S. Sze, Mappings on matrices: invariance of functional values of matrix products, J. Aust. Math. Soc. 81 (2006), no. 2, 165-184.

[5] G.-H. Chan, M.-H. Lim, and K.-K. Tan, Linear preservers on matrices, Linear Algebra Appl. 93 (1987), 67-80.

[6] P. M. Cohn, Algebra. Vol. 1, Second edition, John Wiley \& Sons, Ltd., Chichester, 1982.

[7] D. Dolžan and P. Oblak, Idempotent matrices over antirings, Linear Multilinear Algebra Appl. 431 (2009), no. 5-7, 823-832. 
[8] J. S. Golan, The theory of semirings with applications in mathematics and theoretical computer science, Pitman Monographs and Surveys in Pure and Applied Mathematics, 54. Longman Scientific \& Technical, Harlow; copublished in the United States with John Wiley \& Sons, Inc., New York, 1992.

[9] U. Hebisch and H. J. Weinert, Semirings: algebraic theory and applications in computer science, translated from the 1993 German original. Series in Algebra, 5. World Scientific Publishing Co., Inc., River Edge, NJ, 1998.

[10] N. Jacobson, Lectures in Abstract Algebra. Vol. II. Linear algebra, D. Van Nostrand Co., Inc., Toronto-New York-London, 1953.

[11] S. Kirkland and N. J. Pullman, Linear operators preserving invariants of nonbinary Boolean matrices, Linear Multilinear Algebra 33 (1993), no. 3-4, 295-300.

[12] S. Liu, Linear maps preserving idempotence on matrix modules over principal ideal domains, Linear Algebra Appl. 258 (1997), 219-231.

[13] S.-Z. Song, K.-T. Kang, and L. B. Beasley, Idempotent matrix preservers over Boolean algebras, J. Korean Math. Soc. 44 (2007), no. 1, 169-178.

IMFM

Jadranska 19, 1000 Ljubljana, Slovenia

E-mail address: marko.orel@fmf.uni-lj.si 